\title{
CIRCUMSTELLAR MOLECULAR GAS OF THE YOUNG STELLAR OBJECT SVS 12
}

\author{
KARL R. STAPELFELDT \\ University of Massachusetts, Amherst
}

\section{OVERVIEW}

SVS 12 is an embedded infrared source which is the probable exciting star for HH 12. The source has a "class I" IRAS spectrum and about $20 \mathrm{~L}_{\odot}$ luminosity. Differential reddening between the star $(\mathrm{J}-\mathrm{K} \geq 6 \mathrm{mag})$ and the adjacent infrared reflection nebula ( $\mathrm{J}-\mathrm{K}=3 \mathrm{mag}$ ) suggests the presence of dense circumstellar material (Stapelfeldt et al. 1991). SVS 12 was observed with the OVRO interferometer in the ${ }^{13} \mathrm{CO}$ 1-0 and CS 2-1 lines and associated continuum, and at the CSO with a bolometer and in the CS 5-4 and CS 7-6 lines.

\section{RESULTS}

Bright ${ }^{13} \mathrm{CO}$ emission is distributed in a north-south elongated structure at least $30^{\prime \prime}(10,000 \mathrm{AU})$ in size and which extends beyond the interferometer field of view. Radial velocity changes smoothly from north to south within this structure $\left(\Delta V=2.5 \mathrm{~km} \mathrm{~s}^{-1}\right)$, suggesting rotation. The morphology is rather different in $\mathrm{CS}$, with a single bright clump associated with the northern ${ }^{13} \mathrm{CO}$ peak and a secondary peak amid $\mathrm{HH} 12$. The $\mathrm{CS}$ and ${ }^{13} \mathrm{CO}$ kinematics are identical in the regions where they spatially overlap. No high-velocity gas characteristic of outflow was seen in these lines within the velocity range $\pm 40 \mathrm{~km} \mathrm{~s}^{-1}$.

No continuum emission from SVS 12 was detected at either $2.7 \mathrm{~mm}$ or 3.0 $\mathrm{mm}$; however, an apparently unrelated source is detected $30^{\prime \prime}$ to the NE. At the CSO SVS 12 is strongly detected at $1.1 \mathrm{~mm}, 0.8 \mathrm{~mm}$, and $0.6 \mathrm{~mm}$, with a spectral index characteristic of thermal dust emission.

\section{DISCUSSION}

Using $\mathrm{T}=40 \mathrm{~K}$ (derived from the measured CS 7-6/5-4 line ratio), the observed ${ }^{13} \mathrm{CO}$ flux of $18.5 \mathrm{Jy} \mathrm{km} \mathrm{s}^{-1}$ corresponds to a mass of $0.8 \mathrm{M}_{\odot}$. The CS 2-1 line flux (11 Jy km s$~^{-1}$ ) implies a mass of $0.65 \mathrm{M}_{\odot}$ (Nakamura et al. 1991). For a $\beta=2$ dust opacity the mass implied by the $1.1 \mathrm{~mm}$ CSO continuum flux is $1.0 \mathrm{M}_{\odot}$. There are thus three independent indications of a substantial mass of material within $5000 \mathrm{AU}$ of SVS 12. A toroid is the simplest explanation for the ${ }^{13} \mathrm{CO}$ velocity structure. Some kind of gravitational binding of this system is needed, for the observed velocity gradient would disperse the system in a few times $10^{4}$ years. If we observe the system from near the orbital plane (as the flattened morphology suggests), the velocity gradient requires a dynamical mass of $5 \mathrm{M}_{\odot}$. 
SVS 12: ${ }^{13} \mathrm{CO} 1-0$ and $\operatorname{CS~} 2-1$

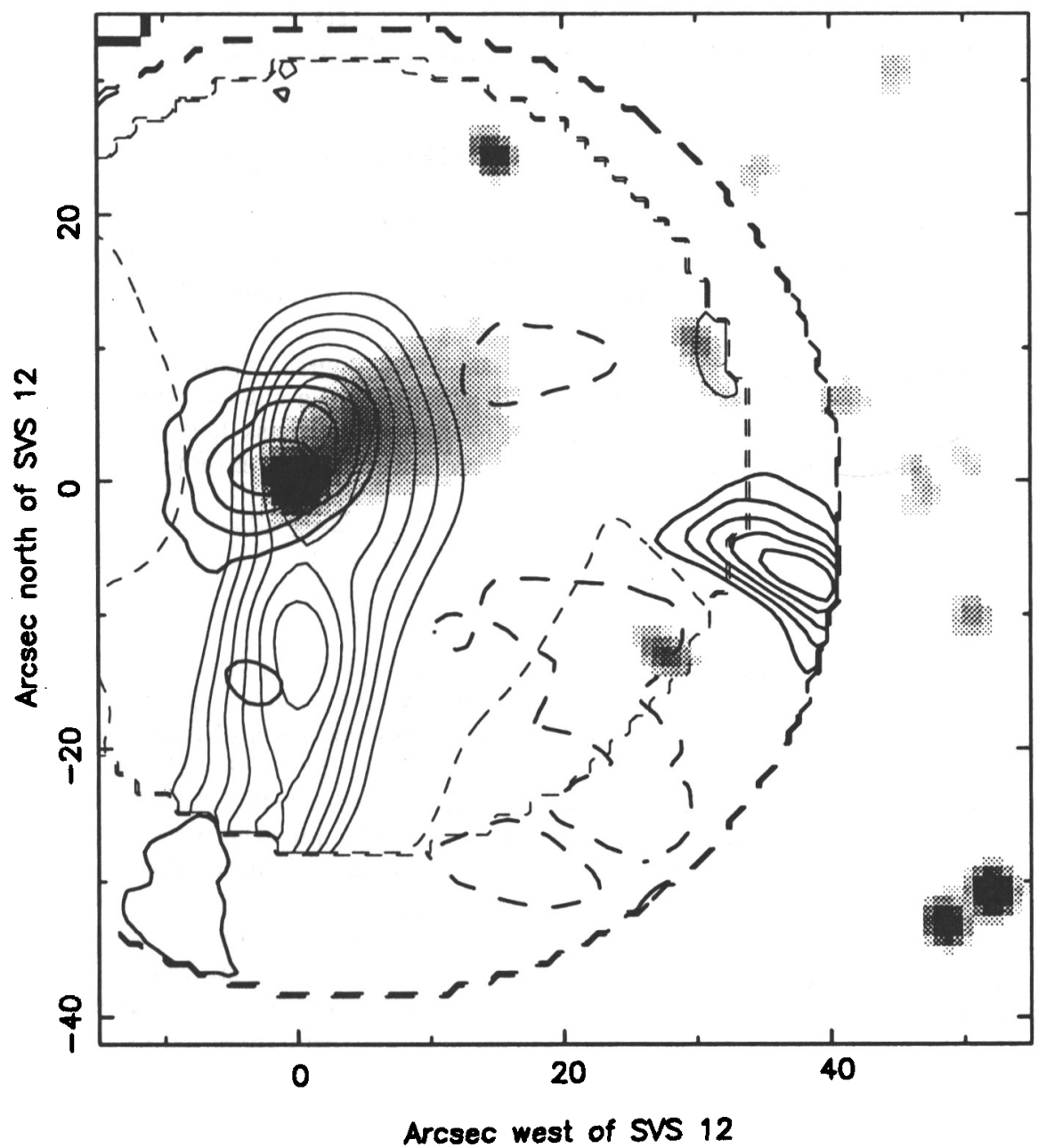

Figure 1: A three-plane overlay showing the CS map (dark contours), the ${ }^{13} \mathrm{CO}$ map (light contours), and a near-infrared image at $2.42 \mu \mathrm{m}$ (greyscale). The large dotted circles indicate the maximum field of view (half-power radius) of the two OVRO maps.

\section{REFERENCES}

Nakamura, A. et al. 1991 ApJ, 383, L81

Stapelfeldt, K. et al. $1991 \mathrm{ApJ}, 371,226$ 